\title{
Transcriptome-based identification of enzymes involved in amino acid biosynthesis in the small brown planthopper, Laodelphax striatellus
}

This article was published in the following Dove Press journal:

Open Access Insect Physiology

20 September 2012

Number of times this article has been viewed

\section{Lu Yang ${ }^{1,2}$ \\ Qiang $\mathrm{Fu}^{2}$ \\ Wen-Bin $\mathrm{Hu}^{\prime}$ \\ Fei $\mathrm{Li}^{\prime}$ \\ Guoqing $\mathrm{Li}^{\prime}$}

'Education Ministry Key Laboratory of Integrated Management of Crop Diseases and Pests, Nanjing Agricultural University, Nanjing, China; ${ }^{2}$ China National Rice Research Institute, Hangzhou, China
Correspondence: Guoqing Li Education Ministry Key Laboratory of Integrated Management of Crop Diseases and Pests, College of Plant Protection, Nanjing Agricultural University, WeiGang No. I, Nanjing 210095, Jiangsu province, China Tel/Fax +86 2584395248 Email liguoqing001234@yahoo.com.cn

\begin{abstract}
The small brown planthopper, Laodelphax striatellus, is a phloem-feeding pest in many important crop plants such as rice and wheat in China. Phloem sap is rich in simple sugars, but low in nitrogenous organic compounds and especially low in essential amino acids. It would be of interest to determine whether this unbalanced diet is compensated for by intracellular microbial symbionts. Based on the transcriptome of L. striatellus and reverse transcription-polymerase chain reaction, we identified 23 fragments that encoded 17 enzymes contributing to amino acid biosynthesis. Sequence alignment, codon usage bias, and phylogenetic analysis indicate that three genes originate from symbiont and the remainder from insect host. It is suggested that enzymes from L. striatellus are responsible for amino group donor synthesis, ammonium assimilation, and biosynthesis of nine amino acids, including glutamate, glutamine, proline, aspartate, asparagine, alanine, serine, glycine, and tyrosine. The biosynthesis of methionine might be catalyzed by enzymes from both host insect and symbiont. Symbiont enzymes are implied to be involved in the biosynthesis of valine, leucine, isoleucine, lysine, and tryptophan. The enzymes catalyzing the syntheses of arginine, threonine, phenylalanine, histidine, and cysteine are not found. Our results imply that the symbionts may partially compensate for the amino acid requirements of $L$. striatellus.
\end{abstract}

Keywords: L. striatellus, amino acid, biosynthesis, enzyme, symbiont

\section{Introduction}

The small brown planthopper, Laodelphax striatellus, is a phloem-feeding pest in many important crop plants such as rice and wheat in China. In general, phloem-feeding pests, such as the pea aphid Acyrthosiphon pisum, lack the capacity for de novo synthesis of ten protein amino acids, namely histidine (His), isoleucine (Ile), leucine (Leu), lysine (Lys), methionine (Met), phenylalanine (Phe), threonine (Thr), tryptophan (Trp), valine (Val), and arginine (Arg), ${ }^{1-3}$ and typically must absorb them from their food. These amino acids are called essential. However, phloem sap is rich in simple sugars, but low in nitrogenous organic compounds and especially low in essential amino acids. In rice phloem sap, for example, sucrose and amino acids are $17 \%-25 \%$ and $3 \%-8 \%$ $(\mathrm{w} / \mathrm{v})$, respectively. Among the free amino acids, asparagine (Asn) (17\%-33\% on a molar basis), glutamate (Glu) (6\%-14\%), serine (Ser) (10\%-13\%), glutamine (Gln) $(7 \%-15 \%)$, Thr $(5 \%-6 \%)$, and Val $(6 \%-7 \%)$ dominate. ${ }^{4}$ Similarly, among the dominant amino acids in wheat phloem sap are also several nonessential amino acids, such as Glu, aspartate (Asp), and Ser. ${ }^{5}$

Intracellular microbial symbionts often compensate for a nutritionally unbalanced diet, ${ }^{6}$ ie, microbial symbionts synthesize essential amino acids for their hosts, and at the 
same time obtain some nonessential amino acids from their hosts. A. pisum, for example, has coevolved with its symbiont Buchnera aphidicola. ${ }^{7}$ Symbiont removal and/or manipulative artificial-diet experiments, ${ }^{8-10}$ coupled with whole-genome sequencing of both Buchnera and A. pisum, ${ }^{1,3,11}$ demonstrate that the biosynthetic capabilities of the holosymbiont (aphid + Buchnera) could meet the nutritional requirements of the host insects. In sum, the association between Buchnera and aphids is an obligate mutualism, which neither can reproduce without the other. ${ }^{12-14}$

Moreover, nitrogen availability is very important to the phloem-feeding insects. Free amino acids are the most important nitrogenous compounds available in phloem sap, predominantly several nonessential amino acids. ${ }^{15}$ In phloem saps of several hosts of $A$. pisum, for example, Asn, Gln, and Glu were dominant. ${ }^{16}$ In addition, $A$. pisum has genes encoding Gln synthetase and Glu synthase. The two enzymes may provide a shuttle for incorporating ammonia into most amino acids through Glu. The two genes are upregulated in bacteriocytes. ${ }^{17}$

In L. striatellus, yeast-like endosymbionts live in the fat body cells. ${ }^{18-20}$ The nutritional importance of the symbionts in compensation for amino acid deficiency remains to be confirmed. The transcriptome of L. striatellus has been published, ${ }^{21}$ and facilitated the identification of the genes encoding putative amino acid synthesis enzymes. The first goal of this study is to identify these genes. Since condon usage bias (CUB) varies both among species and among genes from the same genome, ${ }^{22,23}$ we used CUB and phylogenetic analyses to determine whether these genes are originated from insect host or its symbiont. Our results showed that the symbionts had some important enzymes catalyzing the biosynthesis of Val, Leu, Ile, Lys, and Trp. Whether the symbionts produce these essential amino acids for their host needs further research to confirm.

\section{Materials and methods}

\section{Insect rearing}

L. striatellus nymphs were collected from Nanjing $\left(32.0^{\circ} \mathrm{N}\right.$, $118.5^{\circ} \mathrm{E}$ ), Jiangsu Province, in China in 2007. The strain has been reared routinely on rice, in an insectary under controlled temperature $\left(28^{\circ} \mathrm{C} \pm 1^{\circ} \mathrm{C}\right)$, photoperiod (16 hours light/8 hours dark), and relative humidity (more than $80 \%$ ) since then, with wild stock injections every summer. The rice (Oryza sativa) variety was TN1. Plants were grown in soil at $25^{\circ} \mathrm{C}$ under a long-day photoperiod (16 hours light/8 hours dark) in a growth incubator. The planthoppers were transferred to fresh seedlings every 10-14 days to assure sufficient nutrition.

\section{Retrieval of the genes encoding putative amino acid biosynthesis enzymes}

Reciprocal blast hits were used to search the genes encoding putative amino acid biosynthesis enzymes. The annotated enzymes involved in amino acid biosynthesis from model insects Drosophila melanogaster, Bombyx mori, Anopheles gambiae, Tribolium castaneum, Apis mellifera, and $A$. pisum were performed against the transcriptome data of L. striatellus using the tBLASTn program, ${ }^{24}$ with expectation value less than $1.0 \mathrm{E}-21$. The candidate sequences from $L$. striatellus were reassembled into contigs using CAP3 software and those unassembled remained as singlets, ${ }^{25}$ and then each of the contigs and singlets was compared back to the annotated genomes using BLASTx with default value $(E$-value $\leq 10)$ to determine whether the original sequence was the hit. All the selected contigs and singlets were collected as unigenes. These unigenes were edited and translated into proteins by a user-defined Perl program, Microsoft Excel 2007 (Microsoft, Redmond, WA), and bioedit software ${ }^{26}$ under Linux or Windows.

\section{RNA isolation and cDNA synthesis}

In order to identify key genes involved in biosynthesis of amino acids, three samples mixed with eggs, four to five nymphs (including at least one of the first, second, third, and fourth instars), and sexually mature males and females were collected. The insects were frozen with liquid nitrogen. Total RNA was isolated from the samples following the standard protocol of TRIzol reagent (Life Technologies, Carlsbad, CA). The concentration and quality of total RNA were determined by a NanoDrop spectrophotometer (Thermo Fisher Scientific, Waltham, MA). First-strand cDNA of each sample was synthesized from $1 \mu \mathrm{g}$ of total RNA template using Moloney murine leukemia virus reverse transcriptase (Takara Bio, Otsu, Japan) and an Oligo (dT) 18 primer.

\section{PCR and sequencing}

Reverse transcription-polymerase chain reaction (RT-PCR) was performed to authenticate the sequences of the unigenes. The primers based on the sequences were designed using Primer3 software. ${ }^{27}$ Once initial unigenes from each amino acid biosynthesis enzyme were authenticated, they were aligned to the full sequence of this enzyme from model insect or microorganism species. Some short sequence gaps between two aligned unigenes were found. Specific primers were designed based on the two unigenes between each gap, and the gap was filled by RT-PCR. The final cDNA sequences 
encoding the amino acid biosynthesis enzymes were authenticated using the primers listed in Table 1.

Thermal-cycle programs are available from the authors upon request. Each $50-\mu \mathrm{L}$ PCR reaction contained $2 \mu \mathrm{L}$ of cDNA template, $5 \mu \mathrm{L}$ of $10 \times$ nLA Taq buffer $\left(\mathrm{Mg}^{2+}\right.$-free), $4 \mu \mathrm{L}$ of $\mathrm{MgCl}_{2}(25 \mathrm{mM}), 4 \mu \mathrm{L}$ of deoxyribonucleotide triphosphate mixture ( $2.5 \mathrm{mM} / \mathrm{each}), 2 \mu \mathrm{L}$ of forward and
$2 \mu \mathrm{L}$ of reverse primers $(10 \mu \mathrm{M}), 0.5 \mu \mathrm{L}$ of Taq polymerase (LA Taq, Takara Bio) $(5 \mathrm{U} / \mu \mathrm{L})$, and $32.5 \mu \mathrm{L}$ of doubledistilled $\mathrm{H}_{2} \mathrm{O}$.

Ten- $\mu \mathrm{L}$ PCR products were separated by electrophoresis on $1.2 \%$ agarose gel, stained with ethidium bromide and visualized under ultraviolet light, purified with Wizard DNA Gel Extraction Kit (Promega, Fitchburg, WI), and then cloned

Table I The primers used in reverse transcription-polymerase chain reaction to authenticate the sequences encoding putative enzymes involved in amino acid biosynthesis in Laodelphax striatellus

\begin{tabular}{|c|c|c|}
\hline Putative enzymes & Prime name & Sequence $\left(5^{\prime}-\right.$ to $\left.3^{\prime}-\right)$ \\
\hline \multirow[t]{4}{*}{ Glutamate dehydrogenase, EC I.4.I.3 } & GLDHIF & ATTGCAGTCATAGGACACCG \\
\hline & GLDHIR & CAAAATCGGGGTACAAGAGGT \\
\hline & GLDH2F & ACAGCACGCATAGAACACC \\
\hline & GLDH2R & CCTGGGAAGCCTACAATC \\
\hline \multirow[t]{2}{*}{ Glutamate synthase, EC I.4.I.I3 } & GltSF & AAAAGAAGCGAGCCAAAGACC \\
\hline & GltSR & GGAGGAATCGCCTATGTTTTGG \\
\hline \multirow[t]{2}{*}{ Glutaminase, EC 3.5.1.2 } & GLSF & GTCACCGTTCCGTTCACTCT \\
\hline & GLSR & ATCACCACCAGCATACCTCC \\
\hline \multirow[t]{2}{*}{ Pyrroline-5-carboxylate reductase, EC I.5.I.2 } & P5CRF & TGGCTGTGAAACCCCAATAC \\
\hline & P5CRR & CCATTTTCGCTCCTCCTATC \\
\hline \multirow[t]{2}{*}{ Asparaginase, EC 3.5.I.I } & ASNaseF & CTGTCACGGTCATGGAGGAT \\
\hline & ASNaseR & ACGCTGCGCCAGATTGTAT \\
\hline \multirow[t]{4}{*}{ Aspartate transaminase, EC 2.6.I.I } & ASTIF & GCACAGTCAAACAGAGGGAA \\
\hline & ASTIR & AGAACAGATGATGGCAAACC \\
\hline & AST2F & ATGGGTCCACCAGACGCTAT \\
\hline & AST2R & TCCTCTGTGAATGTTGGACCTGAAT \\
\hline \multirow[t]{4}{*}{ Asparagine synthetase, EC 6.3.5.4 } & ASNSIF & GATTTTGTGCGGATAGGAAC \\
\hline & ASNSIR & GCTATGTGATTGGAGTGCTT \\
\hline & ASNS2F & AGTCAAGGACGGCTATCTCG \\
\hline & ASNS2R & TCACCGTCGAGTCTGTCTTC \\
\hline \multirow[t]{2}{*}{ Saccharopine dehydrogenase, EC I.5.I.I0 } & SDHF & TGCTGCTGTTTTGTCTGCT \\
\hline & SDHR & GCCTAAGATGGCTCGGTAT \\
\hline \multirow[t]{4}{*}{ Alanine transaminase, EC 2.6.I.2 } & ALTIF & GCAGGATTGCCTGGGTTATT \\
\hline & ALTIR & AAAGATTGCCCTGGGAAGAG \\
\hline & ALT2F & ACGATGGTTTGCAGATAGCC \\
\hline & ALT2R & AATCAAGCCTGGAGAAACAC \\
\hline \multirow[t]{4}{*}{ Branched-chain aminotransferase, EC 2.6.I.42 } & BCATIF & CAACATCGGCTTCCTCGTG \\
\hline & BCATIR & CGATCCGGTCGGAACAAC \\
\hline & BCAT2F & CAGAAGCATTCATGCCAATT \\
\hline & BCAT2R & GCCAACGACTGATAAAACAACT \\
\hline \multirow[t]{2}{*}{ Phosphoserine phosphatase, EC 3.I.3.3 } & PSPHF & CCACCGTAACCAATGAAAGC \\
\hline & PSPHR & CCAACATGACAAGATGTGCC \\
\hline \multirow[t]{2}{*}{ Alanine-glyoxylate transaminase, EC 2.6.I.44 } & AGTF & TTGGGCGTGGTTATGACAGC \\
\hline & AGTR & CGGGCATACGAAGCGTTTAT \\
\hline \multirow[t]{4}{*}{ Serine hydroxymethyltransferase, EC 2.I.2.I } & SHMTIF & GATGTGAGTGACGATGAGGGTA \\
\hline & SHMTIR & CGTTCGAGTATGCGGATGTT \\
\hline & SHMT2F & ATTATCGGGCGGCAAAGCAG \\
\hline & SHMT2R & AGGTAACGGGAAGCGTAGCG \\
\hline \multirow[t]{2}{*}{ Cystathionine $\beta$-synthase, EC 4.2.I.22 } & CBSF & GCCCACATAATACCGCCACT \\
\hline & CBSR & ACAGTCCGCAGACCACCATA \\
\hline \multirow[t]{2}{*}{ Cystathionine $\gamma$-lyase, EC 4.4.I.I } & CSEF & CAGTAATGCCCAAAACAGCC \\
\hline & CSER & GGTAATCCGAATCGTGAGGT \\
\hline \multirow[t]{2}{*}{ Tyrosine aminotransferase, EC 2.6.I.5 } & TATF & TCAACGGATATGGTCCCTCA \\
\hline & TATR & CGCATCTTTGTAGTAGCGTCC \\
\hline \multirow[t]{2}{*}{ Tryptophan synthase, EC 4.2.I.20 } & TrpABF & ACTCAGGCAGATGACAACG \\
\hline & TrpABR & GAGCCAACTGGAGGGAAT \\
\hline
\end{tabular}


into pGEM-T easy vector (Promega). The ligation reactions were used to transform Escherichia coli DH5 $\alpha$ competent cells (Tiangen Bio, Beijing, China). Successful strains were screened with blue/white and standard ampicillin selection. Recombinant plasmids were fully sequenced on an Applied Biosystems (Foster City, CA) 3730 automated sequencer.

\section{Calculation of relative synonymous codon usage}

To estimate the CUB among different genes, the relative synonymous codon usage (RSCU) was calculated according to the formula reported previously. ${ }^{22,28}$

$$
\operatorname{RSCU}=\left(\mathrm{g}_{\mathrm{ij}} \times \mathrm{n}_{\mathrm{i}}\right) \div \sum_{\mathrm{j}}^{\mathrm{n}_{\mathrm{i}}} \mathrm{g}_{\mathrm{ij}}
$$

where $g_{\mathrm{ij}}$ is the observed number of the $i$ th codon for $j$ th amino acid that has $\mathrm{n}_{i}$ type of synonymous codons. A codon with an RSCU value of significantly more than 1.0 or less than 1.0 has a positive or a negative CUB. When RSCU value is not significantly different from 1.0, it means that this codon is chosen equally and randomly.

\section{Phylogenetic analysis}

Two candidate enzymes involved in the biosynthesis of amino acids were selected. Their highest hits in other insect species and in microorganisms were collected via BLASTp and tBLASTn $\left(E\right.$-value $\left.<10^{-3}\right)$. These homologues were aligned using ClustalW with default parameters. ${ }^{29}$ The evolutionary history was inferred using the neighbor-joining method..$^{30}$ The bootstrap consensus tree inferred from 1000 replicates is taken to represent the evolutionary history of the taxa analyzed, and the percentage of replicate trees in which the associated taxa clustered together is shown next to the branches. The tree is drawn to scale, with branch lengths in the same units as those of the evolutionary distances used to infer the phylogenetic tree.

\section{Results}

The predicted enzymes involved in amino acid biosynthesis were identified from the transcriptome of the small brown planthopper, L. striatellus, by manual annotation and analysis. In total, 39 unigenes were obtained. These 39 unigenes were assembled and clustered from short reads. There are inevitably issues with clone contamination and mix-up. RT-PCR was performed to prove the correctness of these unigenes using the cDNA template. After short sequence gaps between aligned unigenes were filled by RT-PCR, a total of 23 fragments were obtained; these fragments encoded 17 enzymes that contributed to amino acid biosynthesis. The sequences were submitted to the GenBank database (GenBank accession numbers JQ861711-JQ861735) except that encoding putative tryptophan synthase (EC 4.2.1.20) (shorter than $200 \mathrm{bp}$ ) (Table 2).

\section{Amino group donors and ammonium assimilation}

We cloned cDNA fragments encoding asparaginase (EC 3.5.1.1) and Asp transaminase (EC 2.6.1.1) in L. striatellus. Predicted asparaginase fragment is a truncated protein of 181 aa, and has partial ASRGL1-like domains and dimer interfaces. Moreover, two truncated Asp transaminase fragments were obtained, with lengths of 393 and 178 aa, respectively (Table 2). Asparaginase converted Asn to Asp. Asp transaminase catalyzes amino group transfer from Asp or Glu to a keto acid acceptor such as $\alpha$-ketoglutarate and oxaloacetate. In rice phloem sap, the food of $L$. striatellus, the dominant amino acids are Asn, Glu, Ser, and Gln. ${ }^{4}$ Moreover, Glu, Asp, and Ser were dominant in wheat phloem sap. ${ }^{5}$ Following ingestion, L. striatellus may generate large quantities of Asp and Glu by asparaginase and Asp transaminase and use the two amino acids as metabolic precursors or as amino group donors for the biosynthesis of other amino acids.

Pathways of ammonium assimilation in insects may be via two ways mediated by Glu dehydrogenase (EC 1.4.1.3), Gln synthetase (EC 6.3.1.2), and Glu synthase (EC 1.4.1.13). Glu dehydrogenase catalyzes the reductive amination of $\alpha$-ketoglutarate to yield Glu. Gln synthetase catalyzes the ATP-dependent amidation of the $\gamma$-carboxyl group of Glus to form Gln. Glu synthase catalyzes the reductive amination of $\alpha$-ketoglutarate, using the amide-N of Gln to form two Glus, one from amination of $\alpha$-ketoglutarate and the other from deamidation of Gln. In general, herbivores such as $L$. striatellus feed on green plants that grow under conditions where little ammonium is available, and Gln synthetase and Glu synthase potentially cooperate to incorporate ammonium nitrogen into Glu. ${ }^{17}$ Based on the transcriptome of L. striatellus, we identified predicted Glu dehydrogenase (two truncated fragments with lengths of 421 and 341 aa, respectively) and Glu synthase (445 aa) (Table 2). We need further research to determine whether Gln synthetase exists in L. striatellus or not.

Moreover, a predicted $L$. striatellus cystathionine $\gamma$-lyase (308 aa) (EC 4.4.1.1) was obtained (Table 2). The enzyme 
Table 2 Genes encoding putative enzymes involved in the biosynthesis of protein amino acids in Laodelphax striatellus

\begin{tabular}{|c|c|c|c|c|}
\hline$E C^{a}$ & Unigene $^{\mathrm{b}}$ & Fragment $^{c}$ & Length (aa) & Description $^{d}$ \\
\hline \multicolumn{5}{|c|}{ The $\alpha$-ketoglutarate family (glutamic acid, glutamine, proline, arginine) } \\
\hline I.4.1.3 & 2 & 2 & 421,341 & $\begin{array}{l}\text { The first and the second have } 91 \% \text { and } 53 \% \text { identities to XP_00I95 I } 708 \\
\text { (mitochondrial-like), having complete NAD(P)-binding site on conserved } \\
\text { domain of NAD_bind_I_Glu_DH }\end{array}$ \\
\hline I.4.1.13 & 3 & I & 445 & $\begin{array}{l}\text { 80\% to XP_00I } 948786.2 \text { (NADPH-like), having partial pfam I 3450, } \\
\text { NAD(P)-binding Rossmann-like domain }\end{array}$ \\
\hline 3.5.1.2 & I & I & 218 & $\begin{array}{l}88 \% \text { to } X P \_001601673.2 \text { (Nasonia vitripenns), with nonspecific hit } \\
\text { to PRK0097I glutaminase }\end{array}$ \\
\hline I.5.1.2 & 1 & I & 153 & $\begin{array}{l}43 \% \text { to NP_00I I55857.I, with nonspecific hit to PLN02688, } \\
\text { a pyrroline-5-carboxylate reductase }\end{array}$ \\
\hline \multicolumn{5}{|c|}{ The aspartate family (aspartic acid, asparagine, threonine, lysine, and isoleucine) } \\
\hline 3.5.I.I & I & I & 181 & $\begin{array}{l}\text { 53\% to XP_00I } 944435 . \text { I, having partial ASRGLI-like domains } \\
\text { and dimer interfaces on ASRGLI-like domains }\end{array}$ \\
\hline 2.6.1.1 & 4 & 2 & 393 & $\begin{array}{l}\text { 72\% to XP_002428654.I (Pediculus humanus corporis), } \\
\text { having complete pyridoxal 5'-phosphate-binding site, homodimer } \\
\text { interface, and catalytic residue on AAT-like conserved domain }\end{array}$ \\
\hline & & & 178 & $61 \%$ to XP_00I947848, possessing partial pyridoxal \\
\hline & & & & 5'-phosphate-binding pocket on AAT-like conserved domain \\
\hline 6.3.5.4 & 4 & 2 & 166 & $\begin{array}{l}\text { 36\% to XP_001949447.I, having partial active site on } \\
\text { Gn_AT_II_novel conserved domain }\end{array}$ \\
\hline & & & 318 & $\begin{array}{l}\text { 72\% to XP_001943429.I (glutamine-hydrolyzing-like), } \\
\text { possessing partial active site on AsnB conserved domain } \\
\text { and ligand-binding site on Asn_Synthase_B_C conserved domain }\end{array}$ \\
\hline 1.5.1.10 & 2 & I* & 130 & $\begin{array}{l}85 \% \text { to EFQ35895.I (Glomerella graminicola MI.00I), a saccharopine } \\
\text { dehydrogenase }\end{array}$ \\
\hline \multicolumn{5}{|c|}{ The pyruvate family (alanine, valine, and leucine) } \\
\hline 2.6.1.2 & 6 & 2 & 155,133 & $\begin{array}{l}\text { The first and the second are } 47 \% \text { and } 44 \% \text { to XP_00I9487II.2, both of them have } \\
\text { partial pyridoxal 5'-phosphate-binding pocket on AAT-like conserved domain }\end{array}$ \\
\hline 2.6.1.42 & 2 & $2^{*}$ & 63,51 & $\begin{array}{l}86 \% \text { and } 46 \% \text { to EFY89355.I (Metarhizium acridum CQMa I02), the first has } \\
\text { partial substrate-cofactor-binding pocket and homodimer interface } \\
\text { on conserved domain of BCAT_ } \beta \text { family }\end{array}$ \\
\hline \multicolumn{5}{|c|}{ The 3-phosphoglycerate family (serine, cysteine, glycine, and methionine) } \\
\hline 3.1.3.3 & I & I & 152 & $\begin{array}{l}\text { 6I\% to NP_00I I } 55380.1 \text {, having partial active site and motif II } \\
\text { on HAD-like conserved domain }\end{array}$ \\
\hline 2.6.I.44 & I & I & 238 & $\begin{array}{l}59 \% \text { to XP_001947837.2, having partial pyridoxal 5'-phosphate-binding } \\
\text { pocket on OAT-like conserved domain }\end{array}$ \\
\hline 2.1 .2 .1 & 2 & 2 & 239 & $\begin{array}{l}\text { 64\% to EAW96999.I (Homo sapiens), having partial catalytic residue } \\
\text { and pyridoxal 5'-phosphate-binding pocket on conserved domain of SHMT }\end{array}$ \\
\hline & & & 108 & $\begin{array}{l}55 \% \text { to NP_00I I } 538 \text { I } 2.1 \text {, having nonspecific hit to } \\
\text { cd00378 serine-glycine hydroxymethyltransferase }\end{array}$ \\
\hline 4.2.1.22 & 1 & I & 84 & $\begin{array}{l}69 \% \text { to } X P \_001948 \mid 20.1 \text {, with nonspecific hits to cd0I56I and } \\
\text { TIGROII36, the cysteine synthases }\end{array}$ \\
\hline 4.4.1.1 & 2 & I & 308 & $\begin{array}{l}63 \% \text { to NP_00II } 57405.1 \text {, having partial pyridoxal 5'-phosphate-binding site, } \\
\text { substrate-cofactor-binding pocket, catalytic residue, and homodimer } \\
\text { interface on CGS-like conserved domain }\end{array}$ \\
\hline \multicolumn{5}{|c|}{ The phosphoenolpyruvate and erythrose-4-P family (phenylalanine, tyrosine, and tryptophan) } \\
\hline 2.6.1.5 & 2 & I & 338 & $\begin{array}{l}60 \% \text { to XP_002431054.I (Pediculus humanus corporis), having complete pyridoxal } \\
5^{\prime} \text {-phosphate-binding site and homodimer interface on AAT-like conserved } \\
\text { domain }\end{array}$ \\
\hline 4.2.1.20 & I & I* & 37 & $\begin{array}{l}\text { 84\% to EGS23375.I (Chaetomium thermophilum), having nonspecific hit to } \\
\text { cd06446, a } \beta \text {-tryptophan synthase }\end{array}$ \\
\hline
\end{tabular}

Notes: aSee Table I for the enzyme name of each EC number; 'buigene number found from the transcriptome of Laodelphax striatellus; 'fragment numbers after short sequence gaps between aligned unigenes were filled by reverse transcription-polymerase chain reaction; ${ }^{d}$ percentage means the identity, the encoding proteins $\mathrm{XP} 00195 \mathrm{I} 708$, XP_001948786.2, NP_00II55857.I, XP_001944435.I, XP_001947848, XP_001949447.I, XP_00I943429.I, XP_0032422I0.I, XP_00I9487II.2, NP_00II55380.I, XP_001947837.2, NP_00I I538I2.I, XP_001948I20.I, and NP_00I 157405.I come from Acyrthosiphon pisum genes.

Abbreviations: DH, dehydrogenase; ASRGLI, asparaginase like I; AAT, aspartate aminotransferase; Gn_AT_II, glutamine amidotransferases class-II; AsnB, asparagine synthase (glutamine-hydrolyzing); BCAT, branched-chain amino acid aminotransferase; OAT, ornithine acetyltransferase; SHMT, serine hydroxymethyltransferase; CGS, cystathionine $\gamma$-synthase. 
assimilates 2-oxobutanoate and ammonia into L-cystathionine as part of the Met pathway. This may contribute to Met or cysteine (Cys) production but is not expected to form a significant contribution to the amino acid budget, because cystathionine or Met is not a substrate for biosynthesis of other amino acids.

\section{The $\alpha$-ketoglutarate family of amino acids}

Amino acids derived from $\alpha$-ketoglutarate include Glu, Gln, proline (Pro), Lys, and Arg. The biosynthesis of Glu and Gln were discussed above when we considered pathways of amino group donors and ammonium assimilation. Moreover, a cDNA fragment encoding predicted glutaminase (218 aa) (EC 3.5.1.2) was also cloned from L. striatellus, which is an amidohydrolase enzyme and generates Glu from Gln (Table 2).

Fragment (153 aa) encoding $\Delta 1$-pyrroline-5-carboxylate reductase was cloned from L. striatellus (Table 2). This suggests that Pro is derived from Glu via a series of four reactions catalyzed mainly by $\Delta 1$-pyrroline-5-carboxylate synthetase (EC 2.7.2.11), glutamate-5-semialdehyde dehydrogenase (EC 1.2.1.41), and $\Delta 1$-pyrroline-5-carboxylate reductase (EC 1.5.1.2), just as that in . pisum. $^{1,3,11,31}$

Saccharopine dehydrogenase (EC 1.5.1.10) had transcript in the transcriptome of L. striatellus, which encoded a truncated peptide of 130 aa. This indicated that the metabolism of Lys is via the $\alpha$-aminoadipate pathway, a common route in Saccharomyces cerevisiae and other higher fungi. ${ }^{32}$

\section{The Asp family of amino acids}

The amino acid members of the Asp family include Asp, Asn, Thr, and Ile. Asp and Asn are among the dominant amino acids in rice and wheat phloem sap, the diet of L. striatellus. ${ }^{4,5}$ Moreover, Asp is also formed from oxaloacetate, the citric acid cycle intermediate, by transfer of an amino group from Glu via aminotransferase catalyzed reaction. Asn is also generated by amidation of the $\beta$-carboxyl group of Asp. Asn synthetase (EC 6.3.5.4) catalyzes the ATP-dependent transfer of the amido-N of Gln to Asp to yield Glu and Asn. In addition, asparaginase (EC 3.5.1.1) is an enzyme that catalyzes the hydrolysis of Asn to Asp. The transcripts of Asn synthetase were found in the transcriptome of $L$. striatellus, and the corresponding cDNA fragments were also cloned (Table 2).

Ile is included in the Asp family of amino acids because four of its six carbons derive from Asp via Thr and only two from pyruvate. Discussion of Ile synthesis is presented under the biosynthesis of the pyruvate family of amino acids.

\section{The pyruvate family of amino acids}

Alanine (Ala), Val, and Leu are derived from pyruvate. Transamination between Glu and pyruvate gives Ala. Ala transaminase (EC 2.6.1.2) catalyzes the transamination. Its transcript was found in the transcriptome of L. striatellus and two cDNA fragments (encoding two truncated peptides of 155 and 133 aa, respectively) were cloned (Table 2).

Val, Leu, and Ile are three branched-chain amino acids. Two singlets of the branched-chain amino acid transaminase (EC 2.6.1.42) were found in the transcriptome of L. striatellus, and their cDNA fragments were cloned. These two fragments encoded for two truncated peptides of 63 and 51 aa, respectively. The first peptide had a partial substrate-cofactorbinding pocket and homodimer interface on conserved domain of the BCAT- $\beta$ family (Table 2). Branched-chain amino acid transaminase catalyzes the final reaction in the production of each of the three branched-chain amino acids.

\section{The 3-phosphoglycerate family of amino acids}

Ser, glycine (Gly), Cys, and Met are derived from the glycolytic intermediate 3-phosphoglycerate (3-PG) family. Catalyzed by 3-PG dehydrogenase (EC 1.1.1.95), 3-PG yields 3-phosphohydroxypyruvate, which as an $\alpha$-keto acid is a substrate for transamination by Glu to give 3-phosphoserine. Phosphoserine phosphatase (EC 3.1.3.3) then generates Ser. The transcript of phosphoserine phosphatase was found in the transcriptome of L. striatellus, and 1 cDNA fragment encoding a truncated peptide of 152 aa was cloned (Table 2).

Moreover, Ser hydroxymethyltransferase (EC 2.1.2.1) and Ala-glyoxylate transaminase (EC 2.6.1.44) were predicted and their cDNAs were cloned in L. striatellus (Table 2). This indicated Gly is made via two enzymatic processes. Firstly, Ser hydroxymethyltransferase catalyzes Ser decomposition to form Gly and $\mathrm{N}^{5}, \mathrm{~N}^{10}$-methylenetetrahydrofolate. Secondly, Ala-glyoxylate transaminase catalyzes the transamination between Ala and glyoxylate to generate pyruvate and Gly.

Two orthologues of $D$. melanogaster genes encoding cystathionine $\gamma$-lyase (EC 4.4.1.1) and cystathionine $\beta$-synthase (EC 4.2.1.22) were also cloned in L. striatellus. These enzymes may mediate sequential reactions in the biosynthesis of Met from Cys in L. striatellus (Table 2).

\section{The phosphoenolpyruvate and erythrose- 4-P family of amino acids}

The aromatic amino acids, Phe, tyrosine (Tyr) and Trp, are derived from a shared pathway that has chorismate. 
Chorismate biosynthesis occurs via the shikimate pathway from common intermediates phosphoenolpyruvate and erythrose-4-P.

Phe was biosynthesized from chorismate. Insects generally have the capacity to mediate the terminal reaction in the synthesis of Phe by transaminases (Tyr transaminase (EC 2.6.1.5) and Asp transaminase), and the subsequent transformation of Phe to Tyr via Phe hydroxylase, or Phe 4-monooxygenase (EC 1.14.16.1). The cDNA in L. striatellus encodes a truncated Tyr transaminase of 338 aa, a peptide possessing complete pyridoxal 5'-phosphate-binding site and homodimer interface on AAT-like conserved domain. However, we did not find any cDNA sequence encoding Phe 4-monooxygenase (Table 2).

Synthesis of Trp from chorismate needs Trp synthase (EC 4.2.1.20, TrpAB). A short cDNA fragment probably encoding Trp synthase was cloned in L. striatellus (Table 2).

\section{Codon usage bias of genes encoding amino acid biosynthesis enzymes}

Different codons are used to different extents, and every genome shows particular codon preferences. ${ }^{22}$ Among 17 enzymes in L. striatellus contributing to amino acid biosynthesis, saccharopine dehydrogenase, branched-chain amino acid transaminases, and Trp synthase were similar to those from microorganisms (Table 2); they may be symbiontoriginated and the genes encoding them should show CUB. Therefore, the genes encoding the 17 enzymes involved in amino acid biosynthesis in L. striatellus were separated into two groups: three genes presumably originated from symbiont and remains from the insect host. Their RSCU values were calculated (Table 3).

As expected, there was a marked variation in codon-usage pattern between the two groups. Out of the 64 codons corresponding to the 20 standard amino acids and stop signals, RSCU values of 27 codons from the genes presumably originated from symbiont were more than 1 , and 13 codons had RSCU values of more than 1.5. Among the 27 codons, eleven were $\mathrm{C}$-ended, nine G-ended, five A-ended, and two T-ended. For genes presumably originated from host insect, RSCU values of 29 codons were more than 1 , and five codons had RSCU values of more than 1.5. Among the 29 codons, three were $\mathrm{C}$-ended, four G-ended, ten A-ended and twelve T-ended. It seems that the genes presumably originated from symbiont preferentially used C- and G-ended codons, and those from L. striatellus preferred A- and T-ended ones (Table 3).

\section{Phylogenetic analysis}

To further determine whether the three enzymes were originated from symbiont and the others from host insect, two proteins in each group - branched-chain amino acid aminotransferase and cystathionine $\gamma$-lyase - were selected. Their phylogenetic relationships to corresponding proteins from representative insect species and microorganisms were compared by neighbor-joining trees (Figures 1 and 2).

Branched-chain amino acid aminotransferase from the transcriptome of L. striatellus formed a group with that from A. funigatus, supporting by a $99 \%$ bootstrap replicate, and was far from those originated from Insecta. In contrast, all cystathionine $\gamma$-lyases, including that from the transcriptome of L. striatellus, formed a cluster, and those from the microorganisms formed other clusters (Figures 1 and 2). This result provides another indirect proof to support the claim that some putative enzymes for amino acid biosynthesis in L. striatellus were encoded by symbiont genes.

\section{Discussion}

It is known that $A$. pisum, a phloem-feeding pest, lacks the capacity for de novo synthesis of ten protein-amino acids, namely His, Ile, Leu, Lys, Met, Phe, Thr, Try, Val, and Arg. A primary bacteria endosymbiont, Buchnera aphidicola, is housed inside bacteriocytes of $A$. pisum. ${ }^{7}$ The enzymes encoded by the bacteria genome are responsible for the biosynthesis of Thr, Lys, Trp, Phe, His, and Arg. The other four essential amino acids - Val, Leu, Ile, and Met - were biosynthesized in cooperation with aphid and bacteria enzymes. ${ }^{17,31}$ Thus, the biosynthetic capabilities of the holosymbiont (aphid + Buchnera) were able to meet the nutritional requirements of the host insects. The aphid/Buchnera association is obligate and mutualistic, with neither partner being able to reproduce in the absence of the other. ${ }^{12-14}$

Since aphids have bacterial symbionts, whereas planthoppers have yeast-like symbionts, and comparative studies on amino acid biosynthesis of L. striatellus-symbiont and A. pisum-Buchnera systems are interesting. From the transcriptome of L. striatellus, we identified 23 fragments that encoded 17 enzymes contributing to amino acid biosynthesis. Sequence alignment, CUB, and phylogenetic analyses indicated that three enzymes originate from symbiont and the remains from insect host. It is suggested that enzymes from L. striatellus are responsible for amino group donor synthesis, ammonium assimilation, and biosynthesis of nine nonessential amino acids, including Glu, Gln, Pro, Asp, Asn, Ala, Ser, Gly, and Tyr. The biosynthesis of Met might be catalyzed by enzymes from both host insect and 
Table 3 Relative synonymous codon usage (RSCU) values of the genes for 18 putative enzymes in the synthesis of protein amino acids

\begin{tabular}{|c|c|c|c|}
\hline $\begin{array}{l}\text { Amino } \\
\text { acid }\end{array}$ & Codon & $\begin{array}{l}\text { Genes presumably } \\
\text { from symbiont }\end{array}$ & $\begin{array}{l}\text { Genes presumably } \\
\text { from host }\end{array}$ \\
\hline \multirow[t]{2}{*}{ Phe } & UUU & 0.86 & 0.81 \\
\hline & UUC & 1.14 & 1.19 \\
\hline \multirow[t]{6}{*}{ Leu } & UUA & 0.46 & 0.70 \\
\hline & UUG & 0.69 & 1.57 \\
\hline & CUU & 0.92 & 0.94 \\
\hline & CUC & 2.31 & 0.60 \\
\hline & CUA & 0.23 & 0.68 \\
\hline & CUG & 1.38 & 1.51 \\
\hline \multirow[t]{3}{*}{ Ile } & AUU & 0.53 & 1.19 \\
\hline & AUC & 2.12 & 0.92 \\
\hline & $\mathrm{AUA}$ & 0.35 & 0.89 \\
\hline Met & AUG & 1.00 & 1.00 \\
\hline \multirow[t]{4}{*}{ Val } & GUU & 1.23 & 1.23 \\
\hline & GUC & 1.23 & 0.77 \\
\hline & GUA & 0.31 & 0.52 \\
\hline & GUG & 1.23 & 1.49 \\
\hline \multirow[t]{4}{*}{ Ser } & UCU & 0.00 & 1.09 \\
\hline & UCC & 0.67 & 0.66 \\
\hline & UCA & 0.67 & 1.45 \\
\hline & UCG & 3.67 & 0.81 \\
\hline \multirow[t]{4}{*}{ Pro } & $\mathrm{CCU}$ & 0.86 & 1.09 \\
\hline & $\mathrm{CCC}$ & 0.86 & 0.66 \\
\hline & $\mathrm{CCA}$ & 0.57 & 1.56 \\
\hline & CCG & I.7I & 0.69 \\
\hline \multirow[t]{4}{*}{ Thr } & $A C U$ & 0.00 & 1.36 \\
\hline & ACC & 0.80 & 0.62 \\
\hline & $A C A$ & 1.07 & 1.46 \\
\hline & ACG & 2.13 & 0.56 \\
\hline \multirow[t]{4}{*}{ Ala } & GCU & 1.00 & 1.45 \\
\hline & GCC & 1.50 & 0.93 \\
\hline & GCA & 0.83 & 1.07 \\
\hline & GCG & 0.67 & 0.55 \\
\hline \multirow[t]{2}{*}{ Tyr } & UAU & 1.14 & 1.06 \\
\hline & UAC & 0.86 & 0.94 \\
\hline \multirow[t]{2}{*}{ TER } & UAA & 1.50 & 0.27 \\
\hline & UAG & 0.00 & 0.64 \\
\hline \multirow[t]{2}{*}{$\mathrm{His}$} & CAU & 0.33 & 0.99 \\
\hline & CAC & 1.67 & 1.01 \\
\hline \multirow[t]{2}{*}{ Gln } & CAA & 0.77 & 1.01 \\
\hline & CAG & 1.23 & 0.99 \\
\hline \multirow[t]{2}{*}{ Asn } & AAU & 0.00 & 1.19 \\
\hline & AAC & 2.00 & 0.81 \\
\hline \multirow[t]{2}{*}{ Lys } & AAA & 0.35 & 1.23 \\
\hline & AAG & 1.65 & 0.77 \\
\hline \multirow[t]{2}{*}{ Asp } & GAU & 0.74 & 1.20 \\
\hline & GAC & 1.26 & 0.8 \\
\hline \multirow[t]{2}{*}{ Glu } & GAA & 0.67 & 1.24 \\
\hline & GAG & 1.33 & 0.76 \\
\hline \multirow[t]{2}{*}{ Cys } & UGU & 0.33 & 1.21 \\
\hline & UGC & 1.67 & 0.79 \\
\hline TER & UGA & 1.50 & 2.09 \\
\hline Trp & UGG & 1.00 & 1.00 \\
\hline Arg & CGU & 0.00 & 0.80 \\
\hline
\end{tabular}

(Continued)
Table 3 (Continued)

\begin{tabular}{llll}
\hline $\begin{array}{l}\text { Amino } \\
\text { acid }\end{array}$ & Codon & $\begin{array}{l}\text { Genes presumably } \\
\text { from symbiont }\end{array}$ & $\begin{array}{l}\text { Genes presumably } \\
\text { from host }\end{array}$ \\
\hline \multirow{4}{*}{ Ser } & CGC & 1.60 & 0.48 \\
& CGA & 2.00 & 0.85 \\
\multirow{4}{*}{ arg } & GGG & 0.40 & 0.35 \\
& AGU & 0.33 & 1.22 \\
Gly & AGC & 0.67 & 0.77 \\
& AGA & 0.80 & 2.37 \\
& AGG & 1.20 & 1.14 \\
& GGU & 0.76 & 1.12 \\
& GGC & 1.52 & 1.08 \\
& GGA & 1.71 & 1.45 \\
\hline
\end{tabular}

Note: RSCU represents relative synonymous codon usage values, calculated by summing all three genes presumably originated from symbiont and all 14 genes from insect host.

Abbreviations: Phe, phenylalanine; Leu, leucine; lle, isoleucine; Met, methionine; Val, valine; Ser, serine; Pro, proline; Thr, threonine; Ala, alanine; Tyr, tyrosine; TER, terminal signal; His, histidine; Gln, glutamine; Asn, asparagine; Lys, lysine; Asp, aspartate; Glu, glutamate; Cys, cysteine; Trp, tryptophan; Arg, arginine; Gly, glycine.

symbiont. Symbiont enzymes are implied to be involved in the biosynthesis of Lys, Val, Leu, Ile, and Trp. The enzymes catalyzing the syntheses of Arg, Thr, Phe, His, and Cys were not found.

There are several similarities to amino acid biosynthesis between L. striatellus-symbiont and A. pisum-Buchnera systems. Firstly, both L. striatellus and A. pisum use Asp and Glu as the metabolic precursors and/or amino group donors for the synthesis of other protein amino acids. In rice ${ }^{4}$ and wheat ${ }^{5}$ phloem saps, Asn (Asp) and Glu dominated. In alfalfa phloem sap, the diet of $A$. pisum, Asn comprises approximately $70 \%$ of the total amino acid content. ${ }^{33}$ Following ingestion, both $L$. striatellus and A. pisum convert Asn by the enzyme asparaginase to generate Asp, which is then transaminated to $\alpha$-ketoglutarate by Asp transaminase to liberate Glu. ${ }^{1,31}$

Secondly, the pathway of ammonium assimilation is the same in both $L$. striatellus and A. pisum. In L. striatellus, Gln synthetase and Glu synthase may potentially cooperate to incorporate ammonium nitrogen into Glu. Gln synthetase and Glu synthase are also upregulated in bacteriocytes in A. pisum. ${ }^{17}$ Moreover, the Gln synthetase/Glu synthase cycle has also been characterized in vivo in mosquitoes and silkworms. ${ }^{34-38}$ In mosquitoes, Gln synthetase and Glu synthase are highly expressed in the fat body following a blood meal ${ }^{34}$ and involved in ammonia detoxification. ${ }^{39}$ In silkworms, Glu synthase activation in the posterior silk glands allows enhanced utilization of nitrogen (in the form of Glu converted from Gln) for the synthesis of silk protein. ${ }^{36-38}$ It seems that Gln synthetase/Glu synthase cycle is 


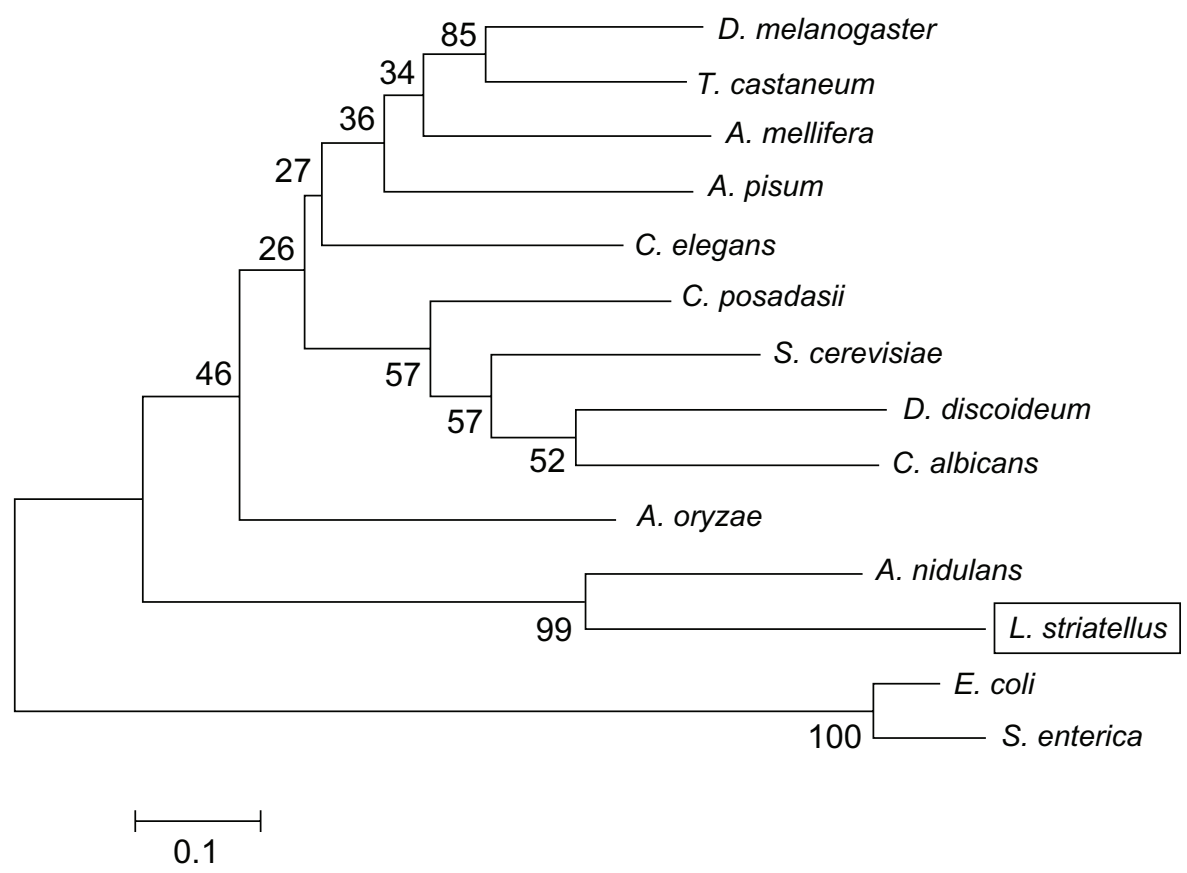

Figure I A phylogenetic tree of branched-chain amino acid aminotransferase in insects and other organisms using the MEGA5 program.

Notes: The sequence from Laodelphax striatellus (L. striatellus) begins at the rectangle. A. mellifera (Apis mellifera, GI: 32879I502), A. pisum (Acyrthosiphon pisum, GI: I936833II), D. melanogaster (Drosophila melanogaster, GI: 2464I779), T. castaneum (Tribolium castaneum, GI: 910776I0), C. elegans (Caenorhabditis elegans, GI: I756860I), S. cerevisiae (Saccharomyces cerevisiae, Gl: 6322002), C. albicans (Candida albicans, GI: 6848278I), D. discoideum (Dictyostelium discoideum, Gl: 66808747), A. nidulans (Aspergillus nidulans, GI: 675I6207), A. oryzae (Aspergillus oryzae, GI: 3I7I57075), C. posadasii (Coccidioides posadasii, Gl: 3033I6402), E. coli (Escherichia coli, GI: 49I76403), S. enterica (Salmonella enterica subsp. enterica serovar Typhi, GI: 16762228).

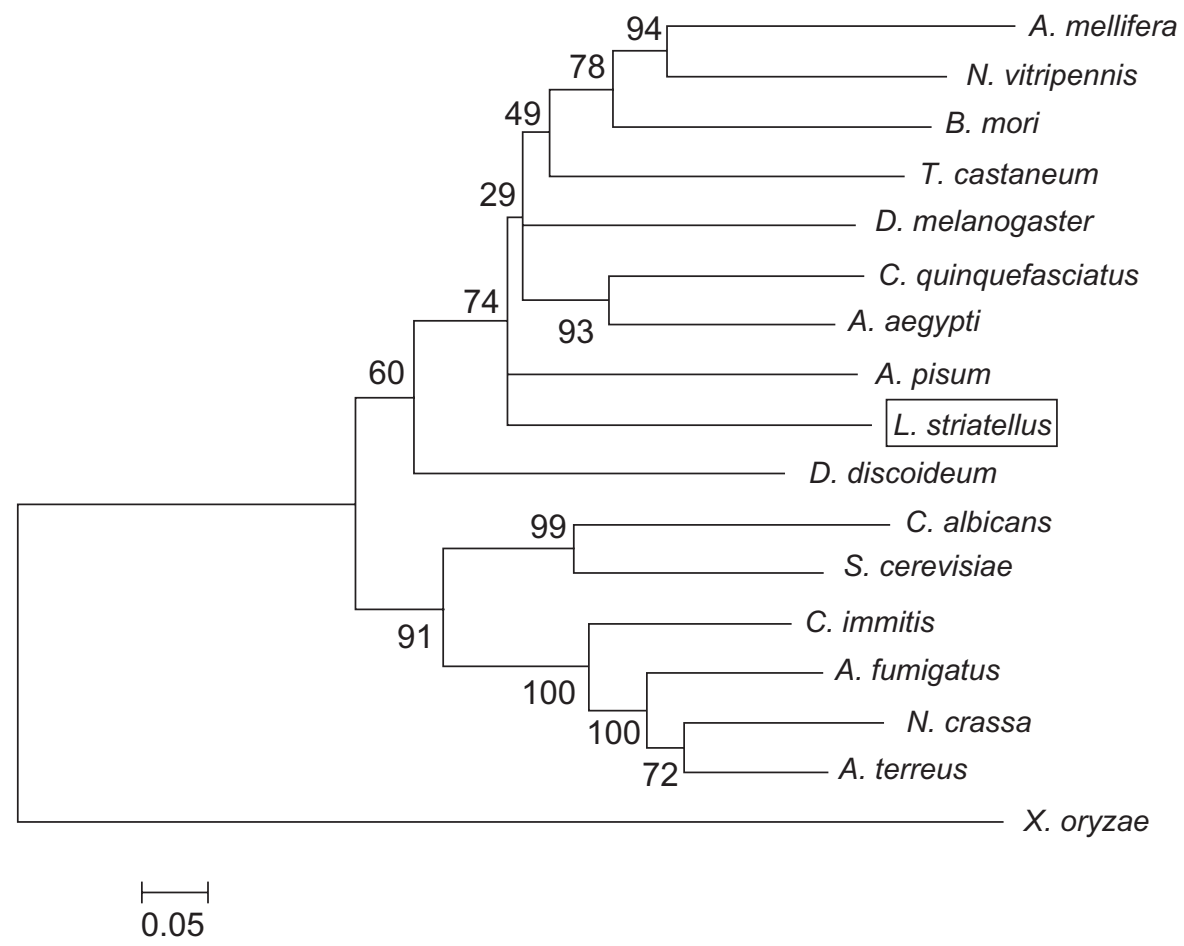

Figure 2 A phylogenetic tree of cystathionine $\gamma$-lyase in insects and other organisms using the MEGA5 program.

Notes: The sequence from Laodelphax striatellus (L. striatellus) begins at the rectangle. A. aegypti (Aedes aegypti, GI: I57I04405), A. pisum (Acyrthosiphon pisum, Gl: 283436226), A. mellifera (Apis mellifera, GI: 66530260), B. mori (Bombyx mori, GI: I I 405 I 238), C. quinquefasciatus (Culex quinquefasciatus, GI: I 70040626), D. melanogaster (Drosophila melanogaster, GI: 19922554), N. vitripennis (Nasonia vitripennis, GI: I5654726I), T. castaneum (Tribolium castaneum, GI: 9I090582), N. crassa (Neurospora crassa, GI: I64423035), A. terreus (Aspergillus terreus, GI: II5491008), A. fumigatus (Aspergillus fumigatus. GI: 70983427), C. immitis (Coccidioides immitis, GI: II9I95678), D. discoideum (Dictyostelium discoideum, GI: 66825335), C. albicans (Candida albicans, GI: 684794I8), S. cerevisiae (Saccharomyces cerevisiae, GI: 4I6I63), X. oryzae (Xanthomonas oryzae pv. Oryzicola, GI: 32493I43). 
an efficient and effective way of ammonium assimilation in many insect species.

In contrast, several differences were also found in amino acid biosynthesis between $L$. striatellus-symbiont and A. pisum-Buchnera systems. Firstly, two pathways have evolved for Lys biosynthesis, the diaminopimelic acid route (DAP) from Asp and the $\alpha$-aminoadipate (AAA) pathway from $\alpha$-ketoglutarate. ${ }^{40}$ Our data indicated that the metabolism of Lys in L. striatellus-symbiont holosymbiont is via the AAA pathway, a common route in Saccharomyces cerevisiae and other higher fungi. ${ }^{32}$ In contrast, Lys in A. pisum was generated by the DAP pathway, subsequently catalyzed by bacterium enzyme Asp kinase (EC 2.7.2.4, ThrA), Aspsemialdehyde dehydrogenase (EC 1.2.1.11, Asd), dihydrodipicolinate synthase (EC 4.2.1.52, DapA), dihydrodipicolinate reductase (EC 1.3.1.26, DapB), 2,3,4,5-tetrahydropyridine-2, 6-carboxylate $N$-succinyltransferase (EC 2.3.1.117, DapD), $N$-succinyldiaminopimelate aminotransferase (EC 2.6.1.17, ArgD), succinyl-diaminopimelate desuccinylase (EC 3.5.1.18, DapE), diaminopimelate epimerase (EC 5.1.1.7, DapF), and diaminopimelate decarboxylase (EC 4.1.1.20, LysA), respectively. ${ }^{1,3,11,31}$

Secondly, branched-chain amino acid transaminase catalyzes the final reaction in the production of three branchedchain amino acids: Val, Leu and Ile. In the A. pisum-Buchnera system, the gene encoding branched-chain amino acid transaminase comes from the host genome. The biosynthesis of Val, Leu, and Ile requires within-pathway metabolic collaboration between symbiotic partner and $A$. pisum. Catalyzed by bacterium acetolactate synthase (EC 2.2.1.6, IlvHI), ketol-acid reductoisomerase (EC 1.1.1.86, IlvC), dihydroxy-acid dehydratase (EC 4.2.1.9, IlvD), and aphid branched-chain amino acid transaminase (ACYPI008372, EC 2.6.1.42), pyruvate was used to generate Val, using Glu as an amino donor. Similarly, pyruvate was applied to synthesize Leu, catalyzed by bacterium IlvHI, IlvC, IlvD, 2-isopropylmalate synthase (EC 2.3.3.13, LeuA), isopropylmalate isomerase (EC 4.2.1.33, LeuCD), 3-isopropylmalate dehydrogenase (EC 1.1.1.85, LeuB), and aphid ACYPI008372. Catalyzed by aphid Thr ammonia lyase (EC 4.3.1.19, ACYPI006784), Thr was transferred to 2-oxobutanoate, and the latter was further transferred to Ile by bacterium IlvHI, IlvC, IlvD, and aphid ACYPI008372. 1,3,31 In the L. striatellus-symbiont system, however, branched-chain amino acid transaminase seems to originate from symbiont. Therefore, the symbionts in L. striatellus were responsible for the biosynthesis of Val, Leu, and Ile.

In summary, our results indicate that amino acid biosynthesis in the L. striatellus-symbiont system is a complex, energy-requiring process, and requires metabolic collaboration between the symbiotic partner and the host. The major weakness of our data is that we used gene fragments, rather than the complete cDNAs in the present paper. This may affect the calculated RSCU values and the phylogenetic tree constructions. We will carry out further experiments to address this issue.

\section{Acknowledgments}

This research was supported by the National Basic Research Program of China (973 Program, no 2010CB126200). We thank Drs Z Han and S Dong of our laboratory for useful discussions during the course of this research.

\section{Disclosure}

The authors report no conflicts of interest in this work.

\section{References}

1. Wilson A, Ashton P, Calevro F, et al. Genomic insight into the amino acid relations of the pea aphid, Acyrthosiphon pisum, with its symbiotic bacterium Buchnera aphidicola. Insect Mol Biol. 2010;19: 249-258.

2. Oliver KM, Degnan PH, Burke GR, Moran NA. Facultative symbionts in aphids and the horizontal transfer of ecologically important traits. Annu Rev Entomol. 2010;55:247-266.

3. Richards S, Gibbs RA, Gerado NM, et al. Genome sequence of the pea aphid Acyrthosiphon pisum. PloS Biol. 2010;8:e1000313.

4. Fukumorita T, Chino M. Sugar, amino acid and inorganic contents in rice phloem sap. Plant Cell Physiol. 1982;23:273-283.

5. Hayashi H, Chino M. Collection of pure phloem sap from wheat and its chemical composition. Plant Cell Physiol. 1986;27:1387-1393.

6. Baumann P. Biology bacteriocyte-associated endosymbionts of plant sapsucking insects. Annu Rev Microbiol. 2005;59:155-189.

7. Baumann P, Baumann L, Lai C, Rouhbakhsh D, Moran NA, Clark MA. Genetics, physiology, and evolutionary relationships of the genus Buchnera: intracellular symbionts of aphids. Annu Rev Microbiol. 1995;49:55-94.

8. Akman Gündüz E, Douglas A. Symbiotic bacteria enable insect to use a nutritionally inadequate diet. Proc Biol Sci. 2009;276:987-991.

9. Douglas A, Prosser W. Synthesis of the essential amino acid tryptophan in the pea aphid (Acyrthosiphon pisum) symbiosis. J Insect Physiol. 1992;38:565-568.

10. Douglas A, Minto L, Wilkinson T. Quantifying nutrient production by the microbial symbionts in an aphid. $J$ Exp Biol. 2001;204:349-358.

11. Shigenobu S, Watanabe H, Hattori M, Sakaki Y, Ishikawa H. Genome sequence of the endocellular bacterial symbiont of aphids Buchnera sp. APS. Nature. 2000;407:81-86.

12. Douglas A. Requirement of pea aphids (Acyrthosiphon pisum) for their symbiotic bacteria. Entomol Expt Appl. 1992;65:195-198.

13. Dunbar HE, Wilson ACC, Ferguson NR, Moran NA. Aphid thermal tolerance is governed by a point mutation in bacterial symbionts. PloS Biol. 2007;5:e96.

14. Montllor CB, Maxmen A, Purcell AH. Facultative bacterial endosymbionts benefit pea aphids Acyrthosiphon pisum under heat stress. Ecol Entomol. 2002;27:189-195.

15. Sandström J, Moran NA. How nutritionally imbalanced is phloem sap for aphids? Entomol Exp Appl. 1999;91:203-210.

16. Sandström J, Pettersson J. Amino acid composition of phloem sap and the relation to intraspecific variation in pea aphid (Acyrthosiphon pisum) performance. J Insect Physiol. 1994;40:947-955. 
17. Hansen AK, Moran NA. Aphid genome expression reveals hostsymbiont cooperation in the production of amino acids. Proc Natl Acad Sci U S A. 2011;108:2849-2854.

18. Noda H. Preliminary histological observation and population dynamics of intracellular yeast-like symbiotes in the smaller brown planthopper, Laodelphax striatellus (Homoptera: Delphacidae). Appl Entomol Zool. 1974;9:275-277.

19. Noda H, Saito T. Histological and histochemical observation of intracellular yeastlike symbiotes in the fat body of the smaller brown planthopper, Laodelphax striatellus (Homoptera: Delphacidae). Appl Entomol Zool. 1977;12:134-141.

20. Noda H, Saito T. The role of intracelular yeastlike symbiotes in the development of Laodelphax striatellus (Homoptera: Delphacidae). Appl Environ Microbiol. 1979;14:453-458.

21. Zhang F, Guo H, Zheng H, et al. Massively parallel pyrosequencingbased transcriptome analyses of small brown planthopper (Laodelphax striatellus), a vector insect transmitting rice stripe virus (RSV). BMC Genomics. 2010;11:303.

22. Grantham R, Gautier C, Gouy M, Mercier R, Pave A. Codon catalog usage and the genome hypothesis. Nucleic Acids Res. 1980;8: r49-r62.

23. Davis JJ, Olsen GJ. Modal codon usage: assessing the typical codon usage of a genome. Mol Biol Evol. 2010;27:800-810.

24. Altschul SF, Madden TL, Schäffer AA, et al. Gapped BLAST and PSI-BLAST: a new generation of protein database search programs. Nucleic Acids Res. 1997;25:3389-3402.

25. Huang X, Madan A. CAP3: a DNA sequence assembly program. Genome Res. 1999;9:868-877.

26. Hall TA. BioEdit: a user-friendly biological sequence alignment editor and analysis program for Windows 95/98/NT. Nucleic Acids Symp Ser (Oxf). 1999;41:95-98.

27. Rozen S, Skaletsky H. Primer3 on the WWW for general users and for biologist programmers. Methods Mol Biol. 2000;132:365-386.

28. Sharp PM, Li WH. Codon usage in regulatory genes in Escherichia coli does not reflect selection for 'rare' codons. Nucleic Acids Res. 1986;14:7737-7749.
29. Thompson JD, Higgins DG, Gibson TJ. CLUSTAL W: improving the sensitivity of progressive multiple sequence alignment through sequence weighting, position-specific gap penalties and weight matrix choice. Nucleic Acids Res. 1994;22:4673-4680.

30. Saitou N, Nei M. The neighbor-joining method: a new method for reconstructing phylogenetic trees. Mol Biol Evol. 1987;4:406-425.

31. Shigenobu S, Wilson ACC. Genomic revelations of a mutualism: the pea aphid and its obligate bacterial symbiont. Cell Mol Life Sci. 2011;68:1297-1309.

32. Urrestarazu L, Borell C, Bhattacharjee J. General and specific controls of lysine biosynthesis in Saccharomyces cerevisiae. Curr Genet. 1985;9:341-344.

33. Girousse C, Bonnemain JL, Delrot S, Bournoville R. Sugar and amino acid composition of phloem sap of Medicago sativa: a comparative study of two collecting methods. Plant Physiol Biochem. 1991;29:41-48.

34. Scaraffia PY, Zhang Q, Thorson K, Wysocki VH, Miesfeld RL. Differential ammonia metabolism in Aedes aegypti fat body and midgut tissues. J Insect Physiol. 2010;56:1040-1049.

35. Scaraffia PY, Zhang Q, Wysocki VH, Isoe J, Wells MA. Analysis of whole body ammonia metabolism in Aedes aegypti using $\left[{ }^{15} \mathrm{~N}\right]$ labeled compounds and mass spectrometry. Insect Biochem Mol Biol. 2006;36:614-622.

36. Hirayama C, Nakamura M. Regulation of glutamine metabolism during the development of Bombyx mori larvae. Biochim Biophys Acta. 2002;1571:131-137.

37. Hirayama C, Saito H, Konno K, Shinbo H. Purification and characterization of NADH-dependent glutamate synthase from the silkworm fat body (Bombyx mori). Insect Biochem Mol Biol. 1998;28:473-482.

38. Shinbo H, Konno K, Hirayama C. The pathway of ammonia assimilation in the silkworm, Bombyx mori. J Insect Physiol. 1997;43:959-964.

39. Scaraffia PY, Isoe J, Murillo A, Wells MA. Ammonia metabolism in Aedes aegypti. Insect Biochem Mol Biol. 2005;35:491-503.

40. Velasco A, Leguina J, Lazcano A. Molecular evolution of the lysine biosynthetic pathways. J Mol Evol. 2002;55:445-449.
Open Access Insect Physiology

\section{Publish your work in this journal}

Open Access Insect Physiology is an international, peer-reviewed, open access journal publishing original research, reports, reviews and commentaries on all areas of insect physiology. The manuscript management system is completely online and includes a very quick and fair peer-review system, which is all easy to use.

\section{Dovepress}

Visit http://www.dovepress.com/testimonials.php to read real quotes from published authors. 